\title{
Análisis de la normativa en Información y Documentación durante la primera década del siglo XXI (2000-2010)
}

An analysis of the 2000-2010 information and documentation standards

Ana Belén Ríos HiLARIo (1), Blanca GIL URDiciain (2)

(1) Departamento de Biblioteconomía y Documentación, Universidad de Salamanca, España, anarihi@usal.es (2) Departamento de Biblioteconomía y Documentación, Universidad Complutense de Madrid, España, mbgil@pdi.ucm.es

\begin{abstract}
Resumen
Se realiza una recopilación y análisis de las normas publicadas en el ámbito de la Información y Documentación durante el periodo comprendido entre los años 2000 y 2010. Tras una breve introducción, en la que se justifica el alcance de la investigación y se definen los objetivos de la misma, se detalla la metodología empleada en la recolección de las normas. Dicha compilación se efectúa a través de la consulta de diversas fuentes de información, que podemos englobar en tres grandes grupos: los organismos de normalización, las webs institucionales de las entidades documentales de titularidad pública y los informes de expertos y profesionales del campo objeto de estudio. Del conjunto de normas obtenidas se examinan de forma detallada las siguientes variables: número de normas por año, normas en función de las áreas definidas, ámbito de aplicación, idioma, traducciones a las lenguas españolas, organismo responsable y tipo de normas.
\end{abstract}

Palabras clave: Información y Documentación. Estándares. Evolución. 2000-2010.

\section{Introducción}

El presente estudio es el resultado de una de las líneas de investigación del grupo "Sistemas de representación y organización del conocimiento" perteneciente al proyecto de investigación $\mathrm{I}+\mathrm{D}+\mathrm{i}$ titulado "La nueva ecología de la información y la documentación en la sociedad del conocimiento: desarrollo de una métrica sistémica, planificación de un observatorio para su seguimiento e identificación de tendencias básicas y retos estratégicos". Dicho proyecto tiene como propósito fundamental "construir un cuadro sistemático que permita dibujar claramente las características y evolución del sector en España, ampliando la discusión en colaboración con los agentes existentes" (2009, p.6).

\begin{abstract}
The standards published in the field of Information and Documentation between 2000 and 2010 have been compiled and analyzed. Three groups of information sources have been used: information from the standardization bodies, websites of public information and documentation entities, and reports by experts and professionals in the field. The resulting set of standards and references was examined in detail according to the following variables: number of standards by year, standards according to the main LIS areas, scope, language, availability of a Spanish translation, responsible agencies and type. The greatest number of standards corresponds to the library area and the main responsible agency is IFLA, but there is a growing number of standards in specialized fields, showing the increasing interdisciplinarity of Information and Documentation. Most of the new standards address the great recent changes in the area, related to the digital information explosion: digital resources, information literacy and the development of new niches.
\end{abstract}

Keywords: Library and information science. Standards. Evolution. 2000-2010.

Una de las misiones encomendadas al citado grupo era la recopilación de la normativa desarrollada durante el periodo comprendido entre el año 2000 y 2010 en el ámbito de la Información y Documentación en general; pero, de modo particular, el análisis se centraría en el examen detallado de la normas correspondientes al área de la representación y recuperación de la información.

El estudio de las normas desarrolladas en nuestro ámbito va a permitir realizar un análisis de la evolución de la disciplina en la pasada década. En una ciencia marcadamente normativa como es la documental el examen de sus pautas se convierte en una labor necesaria. Sobre este hecho conviene hacer la siguiente apreciación: Debido a las características inherentes a la creación de las normas, en ocasiones, se pro- 
duce un desfase entre el surgimiento del fenómeno y la regulación del mismo. De todos modos, el periodo estudiado es lo suficientemente amplio para que los resultados obtenidos demuestren una panorámica bastante representativa de las normas documentales.

A la hora de abordar la investigación, en primer lugar, se procedió a realizar una serie de búsquedas bibliográficas encaminadas a obtener estudios similares que ayudaran a establecer la metodología a seguir. Sin embargo, no se obtuvieron resultados relevantes que satisficieran nuestras demandas. Esto puede deberse a un motivo fundamental. La ejecución de tal empresa se ha podido llevar, como es este caso, a través de proyectos de investigación cuyos resultados no se han hecho públicos $y$, por lo tanto, no se ha podido tener acceso a ellos.

No obstante, sí que se obtuvieron referencias que recogían la normativa publicada por un periodo determinado sobre componentes muy específicos de la Documentación, como por ejemplo, la recopilación de las normas de tesauros realizadas por García Marco (2008); o sobre un organismo normalizado concreto, tal es el caso de la Internacional Standard Organization, Organismo Internacional de Normalización (ISO). En algunos casos se combinaban ambas variables, como por ejemplo el estudio de Arriola Navarrete (2005) sobre la gestión de calidad en las bibliotecas universitarias a través de las normas ISO. Sin embargo, dichos estudios se centraban sobre todo en el análisis del contenido de tales normas más que en su contabilización.

En este punto habría que realizar una breve síntesis de cada una de las publicaciones recopiladas, pero dicha tarea resulta inviable llevarla a cabo en el presente artículo debido a los requisitos inherentes al mismo. En este sentido, se ha dado prioridad al análisis de la pautas más que a los antecedentes y al estado de la cuestión del objeto de estudio. De tal modo, que una de las novedades de la investigación radica también en el establecimiento de la metodología que se desarrollará en el siguiente apartado.

Partiendo de tales premisas el objetivo principal de este estudio es analizar la producción de la normas sobre Información y Documentación desarrolladas durante la etapa 2000-2010. De este objetivo general se derivan a su vez los siguientes objetivos específicos:

1. Detectar cuál ha sido el área documental más prolífica.

2. Averiguar cuál es el organismo normalizador más importante.
3. Comparar el estado de la normalización española con la internacional

\section{Metodología}

Para recolectar la normativa publicada en estos últimos diez años (2000-2010) en el campo de la Información y Documentación, en primer lugar, se establecieron las pautas a seguir en la recopilación de normas.

Para ello inicialmente se fijó la definición del objeto de estudio, es decir, norma o estándar por el que se entiende (ISO/IEC Guide 2:2004):

Documento aprobado por consenso por un organismo reconocido que proporciona reglas, pautas y/o características para uso común, con el objeto de obtener un óptimo nivel de resultados en un contexto dado.

Sin embargo, mientras se recopilaba la normativa se presentó el problema de cómo recolectar la cantidad de estándares de metadatos que han surgido y siguen apareciendo, no sólo en el campo de la Documentación, sino en otras esferas muy diferentes a la de nuestra disciplina. Tras estudiar varias opciones se optó por recopilar aquellos modelos de metadatos directa 0 indirectamente relacionados con el ámbito documental en el sentido más amplio del término.

A la hora de establecer las fuentes a las que a acudir para seleccionar las directrices, se establecieron tres grandes grupos. En primer lugar, se consultó a los organismos productores de las mismas. En este sentido, se puede realizar una diferenciación entre las grandes organizaciones documentales y las agencias de normalización.

En el caso de las organizaciones documentales de carácter internacional destaca, entre sus muchas funciones - como pueden ser la cooperación, investigación y desarrollo dentro de su campo- la creación de la normativa específica de su área. Dos fueron las instituciones más importantes dentro de este grupo: la International Federation of Library Associations and Institutions, Federación Internacional de Asociaciones de Bibliotecarios e Instituciones (IFLA), ejecutora de las directrices para los servicios bibliotecarios; y el Consejo Internacional de Archivos (CIA) encargado de formalizar toda la regulación referente en el área de los archivos.

Como agencias de normalización se acudió primeramente a la ISO, encargada de promover el desarrollo de normas internacionales de fabricación, comercio y comunicación para todas las ramas industriales a excepción de la eléctrica y la electrónica. Su función principal es la de buscar la estandarización de normas de productos y seguridad para las empresas u organizaciones a 
nivel internacional. Posteriormente, se consultó a AENOR (Asociación Española de Normalización y Certificación) que entre sus funciones principales destaca la elaboración de normas técnicas españolas (UNE) con la participación abierta a todas las partes interesadas y la representación de España en los distintos organismos de normalización regionales e internacionales.

En segundo lugar, se accedió a las webs institucionales de los archivos y bibliotecas de titularidad pública, principalmente de ámbito internacional y nacional. En este apartado, se incluyeron aquellas agencias bibliográficas nacionales creadoras de normas aplicables en su territorio, pero que, en ocasiones, como es el caso de la Library of Congress (LC), transcienden de su ámbito local y se convierten en normas internacionales. Por otro lado, en nuestro país, debido al crecimiento en los últimos años de pautas regionales, se decidió acudir también a dichas entidades de carácter autonómico. En este apartado, se incluyen igualmente aquellas páginas profesionales que cuentan con un apartado destinado a la sistematización de normativa y legislación. Así, fue muy útil la información que figura en las webs de las asociaciones profesionales. Destaca en este sentido el Col.legi Oficial de Bibliotecaris-Documentalistes de Catalunya, encargado de la traducción al catalán de la normativa de carácter internacional.

Finalmente, se procedió a establecer encuentros con expertos que colaboraron en las tareas de búsqueda. Es de agradecer especialmente la colaboración prestada tanto por profesionales como docentes.

La consulta a cada una de las fuentes anteriormente citadas no siempre dio como resultado la obtención de las normas. En ocasiones, hubo que acudir a intermediarios, ya que el acceso a la web era restringido, tal es el caso de la normativa del CIA, que se adquirió a través de la página de la Subdirección General de Archivos Estatales. En el caso de AENOR, se estableció contacto directo con ellos tramitando una solicitud de información referente a la normativa objeto de demanda. Otra situación similar se dio con los organismos autonómicos. En este caso, en la mayoría de las ocasiones debido a la falta de información de este tipo en las páginas consultadas, fue necesario ponerse en contacto con estos centros a través del correo electrónico.

A medida que se recopilaban las normas se plantearon los siguientes problemas. El primero de ellos fue si recopilar todas las normas independientemente del idioma o seleccionar una serie de lenguas. Finalmente se eligió esta últi- ma opción, seleccionando todas las normas producidas en inglés y en castellano al igual que las realizadas por cualquier otra lengua española, es decir, en catalán, euskera o gallego.

En esta segunda fase del proyecto, una vez recopilada la normativa se procedió a su clasificación para posteriormente pasar a tabular los datos con objeto de obtener datos cuantitativos fiables. Para ello se estableció un sistema de clasificación que en un primer momento permitió organizar las normas en función de las siguientes grandes áreas: bibliotecas, archivos, documentación y museos. Posteriormente, se pasó a clasificar las normas atendiendo a su propia definición. A la vez que se iba sistematizando cada norma, se revisaban nuevamente las mismas con el objeto de evitar duplicados y obtener nuevas referencias.

Posteriormente, se realizaron una serie de figuras y tablas teniendo en cuenta las siguientes variables:

- Número de normas por año

- Normas en función de las áreas definidas

- Ámbito de aplicación

- Idioma

- Traducciones a las lenguas españolas

- Organismo responsable

- Tipo de normas

La tabulación de los datos se realizó a través de la hoja de cálculo Excel del paquete informático Microsoft 2010, y sus resultados se detallan seguidamente. Finalmente, conviene indicar que por cuestiones de espacio es imposible incluir las referencias completas de cada una de las normas recopiladas, que se publicarán en el informe final del proyecto anteriormente mencionado.

\section{Resultados}

Una vez seleccionadas las normas, y tras la revisión posterior con el objeto de evitar duplicados, en total se recopilaron 188 nomas y estándares cuyo estudio pormenorizado se realiza a continuación de acuerdo con las variables anteriormente definidas.

Si se analiza la evolución normativa a lo largo del periodo de estudio (2000-2010), se comprueba como es el año 2007 el más fructífero, con 27 normas publicadas, frente al 2003 fecha en la que tan sólo se aprueban 8 . El resultado obtenido se puede calificar de muy productivo si se tiene en cuenta que, de promedio, se realiza- 
ron 11 normas por año. En general, la media de ejecución de una norma que regula un determinado hecho suele ser de unos 5 años aproximadamente desde el momento en que se decide la creación de la misma hasta su aprobación definitiva. En algunos casos, este periodo puede alargarse hasta 10 años $y$, en ocasiones, el proyecto se plasma tan sólo en un borrador pendiente de su aprobación definitiva.

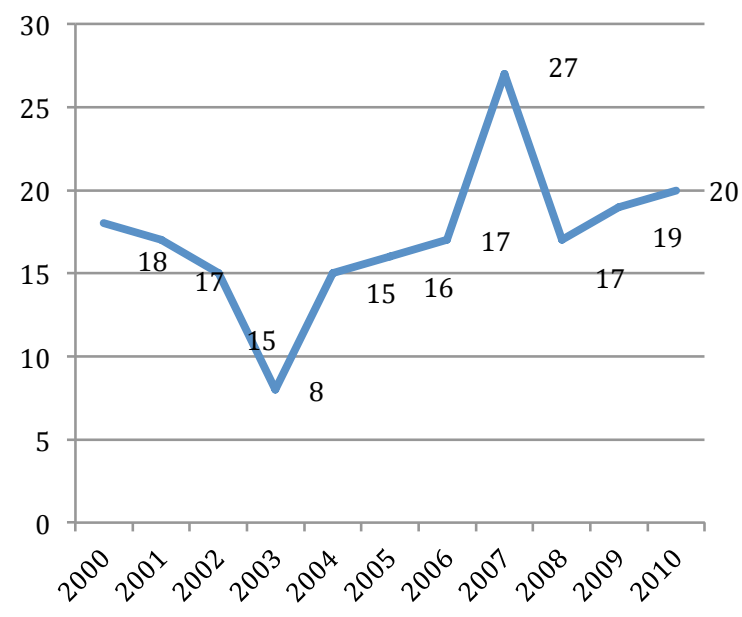

Figura 1. Normas por año

Atendiendo a las grandes áreas o bloques definidos en el apartado metodológico, se puede observar (tabla I) que las normas bibliotecarias son las más numerosas con 78 pautas. En segundo, lugar estarían las normas relacionadas con diversos ámbitos de la Información y Documentación de modo general que hemos englobado bajo el apartado Otros, es decir, aquellas normas que no se corresponden con ninguna de las otras categorías definidas. También es significativo el número de normas publicadas en el ámbito archivístico en estos últimos diez años, el cual ha experimentado un aumento considerable si se compara con otros periodos anteriores. Por otro lado, en el caso de los museos lo más reseñable no es el número de normas, sino que éstas guardan relación directa con las otras áreas documentales. Este hecho pone de manifiesto la estrecha colaboración que se está produciendo en los últimos años entre bibliotecas, archivos y museos y que ha dado lugar a la creación de un nuevo acrónimo - LAM (library, archive and museum) - que engloba a todas estas instituciones.

\begin{tabular}{lr}
\hline Áreas & Número \\
\hline Bibliotecas & 78 \\
\hline Archivos & 30 \\
\hline Documentación & 14 \\
\hline Museos & 6 \\
\hline Otros & 60 \\
\hline Total & 188 \\
\hline
\end{tabular}

Tabla I. Normas por áreas

El ámbito de aplicación de las normas (tabla II) es mayoritariamente internacional con un 94,4 $\%$. Sin embargo, también es destacable la producción de normas de carácter autonómico. Este tipo de pautas son, generalmente, normas de descripción archivísticas.

\begin{tabular}{lr}
\hline Ámbito & Número \\
\hline Internacionales & 170 \\
\hline Nacionales & 7 \\
\hline Autonómicas & 11 \\
\hline Total & 188 \\
\hline
\end{tabular}

Tabla II. Ámbito de aplicación

En relación directa con el anterior apartado está la lengua de publicación de la normativa (tabla III). El idioma mayoritario es el inglés, pero también es reseñable la realización de normas del ámbito documental en varias de las lenguas españolas, en concreto, en castellano, catalán y euskera.

\begin{tabular}{lr}
\hline Lengua & Número \\
\hline Inglés & 153 \\
\hline Castellano & 27 \\
\hline Catalán & 6 \\
\hline Euskera & 2 \\
\hline Total & 188 \\
\hline
\end{tabular}

Tabla III. Lengua

De las normas internacionales se han traducido un total de 31 normas, de las cuales 22 han sido traducidas al castellano y 9 al catalán. Conviene destacar el esfuerzo realizado por las siguientes instituciones a la hora de llevar a cabo tal tarea: 
Biblioteca Nacional de España, Subdirección General de Archivos y Biblioteca Nacional de Cataluña -en colaboración en ocasiones con el Col-legi Oficial de Bibliotecaris-Documentalistes.

\begin{tabular}{lr}
\hline Organismo responsable & Número \\
\hline IFLA & 57 \\
\hline ISO & 15 \\
\hline LC & 16 \\
\hline W3C & 10 \\
\hline UNE & 5 \\
\hline ICA & 6 \\
\hline Getty & 6 \\
\hline Junta de Castilla y León & 4 \\
\hline National Archives of Australia & 3 \\
\hline AES & 3 \\
\hline DC & 3 \\
\hline BNE & 3 \\
\hline Subdirección General de Archivos & 2 \\
\hline Estatales & 2 \\
\hline Subdirecció General d'Arxius i Gestió & 2 \\
\hline Documental. & 2 \\
\hline OcLierno de Aragón & 2 \\
\hline Open Archives Iniciative & 2 \\
\hline Society of American Archivists & 2 \\
\hline
\end{tabular}

Tabla IV. Organismo responsable

En la tabla anterior (tabla IV) se recogen los organismos encargados de realización de las normas. Por razones prácticas, en dicha tabla figuran todas aquellas instituciones responsables de más de una norma. Se pueden clasificar las instituciones en dos grandes grupos: los organismos normativos y las administraciones públicas documentales. En el primer grupo sobresale, muy por encima del resto, la IFLA, autora de 57 de las 188 normas recopiladas. También merece especial atención la labor desempeñada en los últimos años por el W3C, comunidad que desempeña un papel fundamental en el desarrollo de la Web Semántica. En el segundo grupo destacan las instituciones archivísticas de diversos ámbitos. Finalmente, es importante señalar que muchas instituciones se crean por y para el desarrollo de forma exclusiva de un determinado esquema, tal es el caso de $\mathrm{Du}$ blin Core.

En último lugar, se clasifican las normas según su tipología (tabla V).

\begin{tabular}{lr}
\hline Tipo & Número \\
\hline Metadatos & 27 \\
\hline Lenguajes informáticos & 23 \\
\hline Formatos & 21 \\
\hline Tareas y servicios documentales & 18 \\
\hline Descripción de archivos & 16 \\
\hline Modelos conceptuales & 15 \\
\hline Vocabularios controlados & 13 \\
\hline Control de autoridades & 11 \\
\hline Descripción de bibliotecas & 10 \\
\hline Tecnologías de la información & 8 \\
\hline Profesión & 8 \\
\hline Preservación y conservación & 5 \\
\hline Protocolos & 4 \\
\hline Códigos de catalogación & 188 \\
\hline Catálogos & 2 \\
\hline Bibliografías & 2 \\
\hline Instalaciones & 1 \\
\hline Publicaciones oficiales & 2 \\
\hline Total & 13 \\
\hline & \\
\hline
\end{tabular}

Tabla V. Tipología

En este sentido, destacan ligeramente los estándares de metadatos por encima de los lenguajes informáticos - tales como XML-y los formatos -como, por ejemplo, el MARC 21. De nuevo recalcar las normas de descripción archivística, y subrayar también el número de normas que definen modelos conceptuales. EI desarrollo de este tipo de esquemas puede deberse a la influencia del informe de la IFLA publicado en 1998 bajo el título Functional Requirements for Bibliographic Records (FRBR).

Asimismo, es importante resaltar el número de normas destinadas a los vocabularios controlados y al control de autoridades. Para concluir, conviene indicar la importancia que en los últimos años están teniendo las normas referidas de la preservación y conservación documental. 


\section{Conclusiones}

En el apartado precedente se ha realizado un análisis cuantitativo de la normativa documental publicada durante el periodo 2000-2010. De este examen se pueden resaltar como datos más relevantes los siguientes.

El mayor porcentaje de las normas se corresponde con el área bibliotecaria aunque también es cuantioso el número de pautas que guardan relación con varias disciplinas afines, lo que pone de manifiesto el carácter cada vez más multidisciplinar de la Documentación.

La lengua predominante es la inglesa pero, frente a este hecho predecible destaca el esfuerzo realizado en los últimos años por ciertas instituciones nacionales y autonómicas a la hora de traducir la normativa internacional a las lenguas españolas.

Como no podía ser de otro modo, es la IFLA el organismo que mayor número de normas ha publicado durante el periodo objeto de estudio.

Finalmente, son los metadatos el tipo de estándar más fructuoso.

Como conclusión a este estudio, habría que enfatizar que la mayor parte de la nueva normativa es resultado de los cambios vertiginosos que se han producido en nuestra profesión en esta última década, entre los que cabe resaltar la proliferación de los recursos y documentos digitales; la aparición de nuevas misiones documentales como pueden ser la alfabetización informacional; y exploración e incursión en nuevos campos de aplicación.

Por otro lado, a lo largo de esta investigación se han detectado una serie de hechos de carácter metodológico que merecen ser reseñados en este apartado final.

En primer lugar, la dispersión de las normas ha dificultado mucho su localización y posterior recuperación. En este sentido, sería conveniente que las instituciones cabeceras del sistema recogieran en sus webs la normativa que les afecta en todos sus niveles tanto internacional como nacional y local.

Asimismo, las instituciones autonómicas deberían destinar un apartado a través del cual se pudiera acceder a su manual de procedimiento. En lo referente a este asunto tan sólo nos hemos encontrado con cuatro bibliotecas regionales que cumplen con esta premisa (Castila y León, Cataluña, Galicia y La Rioja).
Igualmente, sería positivo que cada organismo recogiera las normas y estándares que emplea a la hora de cumplir con su cometido. Sobre esta materia conviene calificar de ejemplar la estructura y contenido que presenta el apartado Publicacions tècniques de la Biblioteca de Catalunya.

Por último, sería deseable una mayor colaboración por parte de las entidades culturales, ya que tras la debida solicitud de información por medios electrónicos para completar los datos de nuestra investigación figura como hecho negativo la baja respuesta obtenida.

\section{Referencias}

Archivos estatales. [Madrid]: Ministerio de Educación, Cultura y Deporte, Secretaría de Estado de Cultura, 2012. http://www.mcu.es/archivos/index.html/ (2012/04/26).

AENOR: Asociación Española de Normalización y Certificación. Madrid: AENOR, 2010 http://www.aenor.es/ (2012/04/26).

Arriola Navarrete, O. (2005). La gestión de calidad en las bibliotecas universitarias: una perspectiva desde las normas ISO. XXXVI Jornadas Mexicanas de Biblioteconomía, Ixtapa, Zihuatanejo (México). [S.I .: s.n.], 2005. http://eprints.rclis.org/bitstream/10760/6844/1/lagesti\%C 3\%B3ndecalidad.pdf (2012/04/26).

Col.legi Oficial de Bibliotecaris-Documentalistes de Catalunya. Barcelona: COBDC, 2012 http:// www.cobdc.org/ (2012/04/26).

Directorio de bibliotecas españolas. [Madrid]: Ministerio de Educación, Cultura y Deporte, Secretaría de Estado de Cultura, 2012. http://directoriobibliotecas .mcu.es/portada.html/ (2012/04/26).

García Alsina, Montserrat (2008). Metodología para la implantación de la gestión del conocimiento a partir de técnicas documentales. // Scire. ISSN 1135-3716. 14:1 (2008) 43-61.

García Marco, Francisco Javier (2008). Las normas de tesauros se ponen al día: vocabularios estructurados para la recuperación de información en el entorno digital. // Anuario ThinkEPI. ISSN 1886-6344.1 (2008) 57-62.

International Council on Archives. Ica.Org [en línea]. Paris: ICA, 2009. http://www.ica.org/(2012/04/26).

IFLA [en línea]: International Federation of Library Associations and Institutions. The Hague: IFLA, 2012. http://www.ifla.org/ (2012/04/26).

ISO: International Organization for Standardization. Geneva: ISO, 2011http://www.iso.org/ (2012/04/26).

ISO/IEC Guide 2:2004: Standardization and related activities -- General vocabulary. Geneva: ISO, 2011http://www.iso.org/ (2012/04/26).

Library of Congress. Washington: Library of Congress, 2012. http://www.loc.gov/index.html/ (2012/04/26).

Enviado: 2012-05-10. Versión corregida: 2012-06-06. Aceptado: 2012-07-02. 